\title{
Effects of Heavy Sea Quarks at Low Energies
}

\author{
Mattia Bruno, ${ }^{1}$ Jacob Finkenrath, ${ }^{2}$ Francesco Knechtli, ${ }^{2}$ Björn Leder, ${ }^{2,3}$ Rainer Sommer ${ }^{1,3}$
}

(ALPHA collaboration)

\begin{abstract}
${ }^{1}$ John von Neumann Institute for Computing (NIC), DESY, Platanenallee 6, 15738 Zeuthen, Germany
${ }^{2}$ Department of Physics, Bergische Universität Wuppertal, Gaußstraße 20, 42119 Wuppertal, Germany

${ }^{3}$ Institut für Physik, Humboldt-Universität zu Berlin, Newtonstraße 15, 12489 Berlin, Germany
\end{abstract}

(Received 13 November 2014; published 10 March 2015)

\begin{abstract}
We present a factorization formula for the dependence of light hadron masses and low energy hadronic scales on the mass $M$ of a heavy quark: apart from an overall mass-independent factor $Q$, ratios such as $r_{0}(M) / r_{0}(0)$ are computable in perturbation theory at large $M$. The perturbation theory part is stable concerning different loop orders. Our nonperturbative Monte Carlo results obtained in a model calculation, where a doublet of heavy quarks is decoupled, match quantitatively to the perturbative prediction. Upon taking ratios of different hadronic scales at the same mass, the perturbative function drops out and the ratios are given by the decoupled theory up to $M^{-2}$ corrections. We verify-in the continuum limit - that the sea quark effects of quarks with masses around the charm mass are very small in such ratios.
\end{abstract}

DOI: $10.1103 /$ PhysRevLett.114.102001

PACS numbers: 12.38.Gc, 12.38.Bx, 14.65.Dw

Introduction.-One usually presumes that the low energy dynamics of QCD, such as the hadron mass spectrum, is rather insensitive to the physics of heavy quarks. One can then work with QCD with just the three or four light quarks in order to understand it [1]. While large $N_{c}$ (color) arguments suggest a general suppression of quark loop effects, and then a particular one for heavy quarks, so far there has not been any nonperturbative investigation determining the typical magnitude of these effects. This is understandable, since lattice gauge theory with heavy quarks generically has enhanced discretization errors and it is a nontrivial task to separate the physical effects from those unwanted errors. It is thus of high interest for the lattice community to understand whether it is already time to include a charm sea quark in the simulations. Note that one has to be precise about the meaning of the decoupling of heavy quarks $[2,3]$. They do leave traces through renormalization, which we discuss below.

The theoretical tool to understand these questions is the low energy effective theory $[3,4]$ describing the physics with one or more heavy quarks decoupled. We denote this theory by decQCD. The leading order effective theory is just QCD with one or more quark flavors less. The gauge coupling $\bar{g}_{\text {dec }}$ and quark masses of decQCD are adjusted such that decQCD (approximately) reproduces the physics of the (more) fundamental theory at an energy sufficiently below the mass of the decoupled quark [5]. This adjustment is referred to as matching.

We consider the situation with $N_{l}$ light quarks and $N_{q}$ quarks in total. Indicating the flavor content $N_{f}$ of the theory by a subscript, the fundamental theory is $\mathrm{QCD}_{N_{q}}$.
The theory with only the light quarks is $\mathrm{QCD}_{N_{l}}$. Hadronic quantities, the couplings, and the $\Lambda$ parameters in these theories are distinguished by subscripts $q$ and $l$.

In this Letter we briefly present the effective theory from the nonperturbative point of view, discuss the perturbative matching of its parameters in terms of renormalization group invariants (RGI) and point out the factorization formula

$$
\frac{m_{q}^{\mathrm{had}}(M)}{m_{q}^{\mathrm{had}}(0)}=Q_{l, q}^{\mathrm{had}} \times P_{l, q}\left(M / \Lambda_{q}\right)+O\left(\left(\Lambda_{q} / M\right)^{2}\right) .
$$

It gives the mass dependence of hadron masses or hadronic scales such as $r_{0}$ [6] or $t_{0}, w_{0}[7,8]$ in terms of two factors. The first factor, $Q_{l, q}^{\text {had }}$, depends on the hadron mass or hadronic scale and involves only information from the theories with $N_{q}$ and $N_{l}$ massless quark flavors [9]. The second factor, $P_{l, q}\left(M / \Lambda_{q}\right)$, gives the relation of the $\Lambda$ parameters of these two theories, determined such that the low energy physics of the fundamental theory, $\mathrm{QCD}_{N_{q}}$ with $N_{q}-N_{l}$ quarks of RGI mass $M$, is the same as the one of $\mathrm{QCD}_{N_{l}}$ up to power corrections $O\left(\left(\Lambda_{q} / M\right)^{2}\right)$. Throughout this Letter we take the $\Lambda$ parameters to be defined in the $\overline{\mathrm{MS}}$ scheme, but this choice is irrelevant; namely, $Q, P$ have a trivial scheme dependence in regular schemes [10]. Interestingly, the asymptotics of $P_{l, q}\left(M / \Lambda_{q}\right)$ for large mass $M$, is computable in perturbation theory. The formula thus provides a factorization into a nonperturbative piece $Q$, and a "perturbative" one. In particular, the mass dependence is perturbative. We here use quotation marks since the precise meaning is that the asymptotics is perturbative.

We further report on our investigation of the numerical precision of perturbation theory for $P$ and then compare 
Eq. (1) to a first nonperturbative investigation for $N_{q}=2, N_{l}=0$, which we expect to be a quite realistic model for real QCD. In this case, the lowest order effective theory is the Yang-Mills theory, as long as we look at the gluonic sector only, which we do here. Finally, we argue through our numerical simulations that the effects of a charm quark, which are missed by simulating just $\mathrm{QCD}$ with $N_{l}$ quarks, are very small in typical ratios of hadronic scales.

The effective theory: decQCD.-The leading order low energy effective theory is $\mathrm{QCD}_{N_{l}}$. Next-to-leading order (NLO) correction terms in the local effective Lagrangian are gauge-, Euclidean-, and chiral-invariant local fields. These invariances allow only for fields $\Phi_{i}(x)$ of at least dimension six [11]. The Lagrangian may then be written as

$$
\mathcal{L}_{\mathrm{dec}}=\mathcal{L}_{\mathrm{QCD}_{N_{l}}}+\frac{1}{M^{2}} \sum_{i} \omega_{i} \Phi_{i}+O\left(M^{-4}\right),
$$

with dimensionless couplings $\omega_{i}$ which depend logarithmically on the mass $M$.

At the lowest order in $1 / M$, a single coupling [12] $\bar{g}_{\mathrm{dec}}$ is adjusted such that the low energy physics of $\mathrm{QCD}_{N_{l}}$ and $\mathrm{QCD}_{N_{q}}$ match for energies $E \ll M$. It then suffices to require one physical low-energy observable to match, e.g., a physical coupling. Discussing the issue in perturbation theory [5], Bernreuther and Wetzel chose the MOM coupling as a physical coupling and worked out the matching of the $\overline{\mathrm{MS}}$ coupling. Meanwhile, the matching of the latter is known to high-perturbative order. We use this information below.

For now, we remain with the lowest order theory; i.e., all terms $O\left(E^{2} / M^{2}\right)$ are neglected and the Lagrangian is $\mathcal{L}_{\text {dec }}=\mathcal{L}_{\mathrm{QCD}_{N_{l}}}$. We just make use of the fact that there is a single coupling, the gauge coupling $\bar{g}_{\text {dec }}$. Specifying a renormalization scheme, its $\beta$ function is fixed and the coupling is a unique function $\bar{g}_{\mathrm{dec}}=\bar{g}_{l}\left(\mu / \Lambda_{l}\right)$, where $\mu$ is the renormalization scale. Therefore, the matching condition between $\bar{g}_{\mathrm{dec}}$ and $\bar{g}_{q}$ is equivalent to a relation between the $\Lambda$ parameters. Considering only RGIs, the only additional parameter is the quark mass $M$ of the fundamental theory. Therefore, we have to set

$$
\Lambda_{l}=\Lambda_{\mathrm{dec}}\left(M, \Lambda_{q}\right)
$$

in order to match the two theories. For dimensional reasons the unknown function $\Lambda_{\mathrm{dec}}$ can be written as

$$
\Lambda_{\mathrm{dec}}\left(M, \Lambda_{q}\right)=P_{l, q}\left(M / \Lambda_{q}\right) \Lambda_{q} .
$$

In general the $\Lambda$ parameter of an asymptotically free theory is a free, dimensionful, constant, which is to be fixed from outside, usually by matching the theory to experiment. In the present case, experiment for $\mathrm{QCD}_{N_{l}}$ is replaced by $\mathrm{QCD}_{N_{q}}$, where the overall energy scale $\Lambda_{q}$ remains free as before.
The factorization Eq. (1) is a simple consequence of Eq. (4): consider low energy scales of the theory, in particular, hadron masses $m^{\text {had }}$. After matching (and neglecting terms of order $\Lambda_{q}^{2} / M^{2}$ ) they are equal in the fundamental and in the effective theory, $m_{l}^{\text {had }}=m_{q}^{\text {had }}$. We note further, that in $\mathrm{QCD}_{N_{l}}$ there are no mass parameters, the only scale is $\Lambda_{l}$ and hence hadron masses are $m_{l}^{\text {had }}=$ $\rho^{\text {had }} \Lambda_{l}$ with pure numbers $\rho^{\text {had }}$. Thus $m_{l}^{\text {had }} / \Lambda_{l}$ is independent of $M$. In the fundamental theory $m_{q}^{\text {had }}(M) / \Lambda_{q}$ does of course depend on $M$, but $\Lambda_{q}$ is by definition independent of $M$. Together these facts entail the relation Eq. (1) with

$$
Q_{l, q}^{\mathrm{had}}=\frac{m_{l}^{\mathrm{had}} / \Lambda_{l}}{m_{q}^{\mathrm{had}}(0) / \Lambda_{q}}
$$

defined entirely through the two massless theories.

Even though the physics of the two theories is matched at energy scales far below the mass, the perturbative matching of the couplings is in fact best done with a renormalization scale $\mu$ of the order of the mass $[3,5]$. Higher order perturbative corrections then vanish asymptotically as $M \rightarrow \infty$ and the matching of the couplings is indeed perturbative. This entails that $P_{l, q}$ can be computed in perturbation theory when the mass is large.

The Bernreuther-Wetzel relation between the $\overline{\mathrm{MS}}$ couplings $\bar{g}_{\mathrm{dec}}=\bar{g}_{l}\left(m_{*} / \Lambda_{l}\right)$ and $\bar{g}_{q} \equiv \bar{g}_{q}\left(m_{*} / \Lambda_{q}\right)$ is meanwhile known to four loops $[13,14]$,

$$
\bar{g}_{\mathrm{dec}}^{2}=\bar{g}_{q}^{2} \times\left[1+c_{2} \bar{g}_{q}^{4}+c_{3} \bar{g}_{q}^{6}+\cdots\right],
$$

where $c_{2}=\left(N_{q}-N_{l}\right) \frac{11}{72}\left(4 \pi^{2}\right)^{-2}$, and $c_{3}=\left[\frac{572437}{62208}-\frac{84185}{13824} \zeta_{3}-\right.$ $\left.\frac{2633}{15552} N_{l}\right]\left(4 \pi^{2}\right)^{-3}$ for $N_{q}-N_{l}=2$, and $c_{3}=\left[\frac{567431}{124416}-\frac{82043}{27648} \zeta_{3}-\right.$ $\left.\frac{2633}{31104} N_{l}\right]\left(4 \pi^{2}\right)^{-3}$ for $N_{q}-N_{l}=1$. In this relation, the $c_{1} \bar{g}_{q}^{2}$ term in the brackets is missing since $c_{1}$ vanishes for our choice of renormalization scale, $\mu=m_{*}$, where $m_{*}$ satisfies $\bar{m}_{\overline{\mathrm{MS}}}\left(m_{*} / \Lambda_{q}\right)=m_{*}$ with $\bar{m}_{\overline{\mathrm{MS}}}$ the quark mass in the $\overline{\mathrm{MS}}$ scheme.

From now on we suppress indices $l, q$ on $\Lambda$ and $\bar{g}$, since the effective theory only appears implicitly through the previously defined quantities $Q, P$. We define a renormalization group invariant mass scaling function by the logarithmic derivative $\left[P^{\prime}(x)=(d / d x) P(x)\right]$

$$
\left.\eta^{\mathrm{M}}(M) \equiv \frac{M}{P} \frac{\partial P}{\partial M}\right|_{\Lambda}=\frac{M}{\Lambda} \frac{P^{\prime}}{P} \stackrel{M \rightarrow \infty}{\sim} \eta_{0}+\eta_{1}^{M} \bar{g}^{2}+\cdots
$$

with respect to the RGI mass $M$. Just like $M$ itself, $\eta^{M}(M)$ is independent of the scheme. Residual dependences only result when it is evaluated approximately, e.g., at a finite order of perturbation theory. We worked out its perturbative expansion [15], using Eq. (6) and the known expansions of the QCD $\beta$ function and the mass anomalous dimension in the $\overline{\mathrm{MS}}$ scheme up to 4-loop [16-19]. Here we only report 


$$
\begin{gathered}
\eta_{0}=1-\frac{b_{0}\left(N_{q}\right)}{b_{0}\left(N_{l}\right)}>0, \\
\eta_{1}^{M}=-\frac{b_{0}\left(N_{q}\right)}{b_{0}\left(N_{l}\right)}\left(\frac{b_{1}\left(N_{q}\right)}{b_{0}\left(N_{q}\right)}-\frac{b_{1}\left(N_{l}\right)}{b_{0}\left(N_{l}\right)}\right)-\frac{\eta_{0}}{2 \pi^{2}},
\end{gathered}
$$

with $b_{0}(n)=(11-2 n / 3) /(4 \pi)^{2}, b_{1}(n)=(102-38 n / 3) /$ $(4 \pi)^{4}$, and refer the reader to [15] for the general expressions and details of the perturbation theory. Integrating Eq. (7) gives an asymptotic expression $[\tau=\log (M / \Lambda)]$

$$
P=\frac{1}{k} \exp \left(\eta_{0} \tau\right) \tau^{\eta_{1}^{M} / 2 b_{0}\left(N_{q}\right)} \times\left(1+\mathrm{O}\left(\frac{\log \tau}{\tau}\right)\right),
$$

where the constant $k$ is fixed by our conventions for the $\Lambda$ parameter and the RGI mass $M$ [20] to $\log k=\left\{b_{1}\left(N_{q}\right) / 2\left[b_{0}\left(N_{q}\right)\right]^{2}\right\} \log 2-\left\{b_{1}\left(N_{l}\right) / 2\left[b_{0}\left(N_{l}\right)\right]^{2}\right\} \times$ $\log \left[2 b_{0}\left(N_{q}\right) / b_{0}\left(N_{l}\right)\right]$. It turns out that in the $\overline{\text { MS scheme }}$ the higher order corrections to $\eta^{M}$ as well as the function $P$ are very small as far as they are known, namely, up to an impressive 4-loop level, $\eta_{3}^{M} g^{6}$. We discuss an example below.

We now turn to a nonperturbative investigation of the question of how well the mass dependence at intermediate masses $M$ matches onto the asymptotic perturbative prediction. For this purpose we simulate a model, namely, QCD with two heavy, mass-degenerate quarks. The effective theory, decQCD, then is the Yang-Mills theory up to $1 / M^{2}$ corrections $\left(N_{q}=2, N_{l}=0\right)$.

In Monte Carlo simulations of QCD with $N_{f}=2$ massdegenerate $O(a)$ improved Wilson fermions [21] we compute hadronic scales, e.g., $r_{0}(M) / a$, at three values of the lattice spacing $a=0.066,0.049$, and $0.034 \mathrm{fm}$. The RGI mass $M$ is obtained along the lines of [22]. For details about the numerical computations, performed with MP-HMC [23], OPENQCD [24], the package MESONS [25], and the methods applied see Refs. [15,26].

For the hadronic scale $r_{0}$ [6], Eq. (1) takes the form $r_{0}(0) / r_{0}(M)=Q \times P(M / \Lambda)+O\left((\Lambda / M)^{2}\right)$ with $Q=$ $\left[\Lambda r_{0}(0)\right]_{N_{f}=2} /\left[\Lambda r_{0}\right]_{N_{f}=0}$. The ratios $r_{0}(M) / r_{0}(0)$ for $N_{f}=$ 2 are plotted in Fig. 1 as a function of $\Lambda /(\Lambda+M)$. The value $r_{0}(0) / a$ in the chiral limit is taken from [22] for $a=0.066$ and $0.049 \mathrm{fm}$, and we estimate it to $13.06(42)$ at $0.034 \mathrm{fm}$.

The red curve in Fig. 1 shows the mass dependence close to the chiral limit as fitted in [22] with the dashed red lines representing the error of the fit. At large $M / \Lambda$ the blue curve in Fig. 1 is drawn using the 2-loop perturbative formula for $P$ in Eq. (10) and $Q=0.789(52) / 0.602(48)=$ $1.30(14)$ known from previous work $[20,22]$. The dashed blue lines represent the uncertainty of $Q$. The dotted black curve is drawn using the 4-loop value of $P$ and shows that higher perturbative orders are very small. They are negligible in comparison to the uncertainty of $Q$. As our present

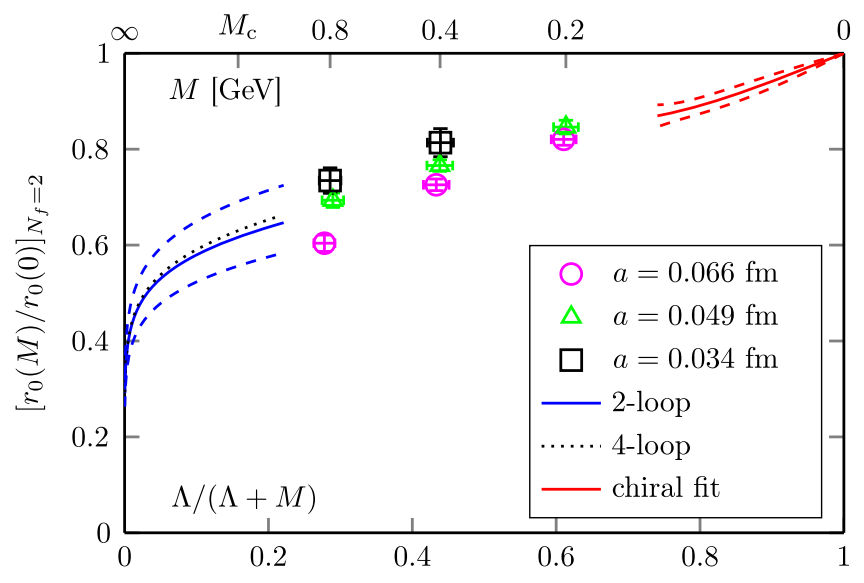

FIG. 1 (color online). The mass dependence of $r_{0}(M) / r_{0}(0)$ in the $N_{f}=2$ theory. Monte Carlo data (symbols) are compared with the perturbative predictions for $1 /(Q P)$ at large $M$. A fit to data close to the chiral limit is also shown.

nonperturbative results, we take the values at the smallest lattice spacing $(a=0.034 \mathrm{fm})$. For $M / \Lambda=2.50$ or $M \approx$ $0.8 \mathrm{GeV}$, a rather modest value of the mass, these are consistent with the (upper error bar of the) factorization curve. Thus within our precision, the perturbative prediction is verified.

By discretizing the derivative in Eq. (7) as $\eta^{M} \approx$ $\log \left[r_{0}\left(M_{2}\right) / r_{0}\left(M_{1}\right)\right] / \log \left(M_{2} / M_{1}\right)$ we obtain from our simulations numerical estimates of $\eta^{M}$. Their values are between 0.12 and 0.17 and are very close to perturbation theory $\eta_{0} \approx 0.12$. A more precise statement needs a careful continuum limit, both for $\eta^{M}$ and in Fig. 1. The lattice community should address this issue in the near future.

Power corrections $O\left(\Lambda^{2} / M^{2}\right)$.- So far we have discussed a comparison of the full theory to the prediction of the factorization formula resulting from the lowest order effective theory. When we take ratios of different hadron masses or different hadronic scales, the function $P(M / \Lambda)$ drops out and we have access to the $O\left(\Lambda^{2} / M^{2}\right)$ power corrections without any perturbative uncertainties. We consider ratios

$$
R=\sqrt{t_{0}} / w_{0}, \quad r_{1} / r_{0}, \quad r_{0} / \sqrt{t_{0}}, \quad \sqrt{t_{c} / t_{0}},
$$

where the scale $t_{c}$ is defined through the smoothed action density [7] $E(t)$ via $t_{c}^{2}\left\langle E\left(t_{c}\right)\right\rangle=c$ with $c=0.2$. It is a shorter distance cousin of $t_{0}$ [7].

We target the mass values $M / \Lambda=0.63,1.28,2.50$, which correspond approximately to $0.2,0.4,0.8 \mathrm{GeV}$. For comparison the RGI charm mass $M_{c} \approx 1.6 \mathrm{GeV}$ [27]. We correct the ratios $R$ for small differences between the targeted and the simulated values of the masses. In the corrections we neglect the error on $M / \Lambda$ since it mainly comes from $\Lambda$ and is therefore common to all points.

Our continuum extrapolations are performed by global fits, 


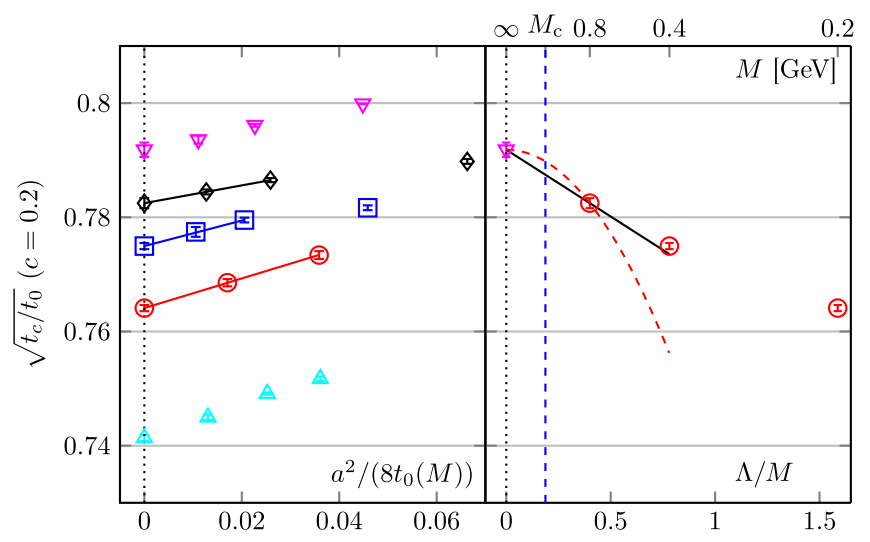

FIG. 2 (color online). Left: The continuum extrapolation of the ratio $\sqrt{t_{c} / t_{0}}(c=0.2)$ at mass values (from top to bottom) $M / \Lambda=\infty, 2.5,1.28,0.63,0$. Right: Its mass dependence including a linear and quadratic interpolation in $\Lambda / M$ between the largest mass and $N_{f}=0(M / \Lambda=\infty)$.

$$
R_{\mathrm{Lat}}=R(M)+s \frac{a^{2}}{8 t_{0}}\left(1+k_{1} \frac{M}{\Lambda}+k_{2} \frac{M^{2}}{\Lambda^{2}}\right),
$$

to all the data. Where it is known, we fix the slope $s$ (which describes the mass independent cutoff effects) from its value determined at $M=0$, cf. [28]. As a representative case, we show in Fig. 2 (left) the global fit for $R=\sqrt{t_{c} / t_{0}}$. The slope $s=0.295$ has been determined from a continuum extrapolation of $\sqrt{t_{c} / t_{0}}$ in the chiral limit (cyan upward-facing triangles). Our fits yield $k_{2}$ compatible with zero. We drop it for our preferred continuum extrapolation, which then gives $k_{1}=-0.19(6)$ and an excellent quality of the fit. The continuum limit values are very precise and allow us to determine the size of the mass effects in the ratio $R$. For comparison, the magenta downward-facing triangles in Fig. 2 are the results for $N_{f}=0$, which according to Eq. (1) is recovered in the limit $M / \Lambda \rightarrow \infty$.

In Fig. 2 (right) we plot the values $R(M)$ (red circles) together with $R(\infty)$ in the $N_{f}=0$ Yang-Mills theory (magenta downward-facing triangle). While the effective theory expectation is a roughly quadratic behavior in $\Lambda / M$, the full theory results are approximately linear in that variable. The natural explanation-since we do not have any doubt about the validity of the effective theory description-is that the masses of our simulations are not yet large enough to be described by NLO decQCD (Yang-Mills plus $1 / M^{2}$ corrections). Taking the largest mass and the $N_{f}=0$ value we can obtain by simple linear interpolation in $1 / M$ (black line) and $1 / M^{2}$ (red dashed line) two estimates of the mass effects at the charm mass marked by the blue vertical dashed line.

The dynamical fermion effects of these heavy quarks are very small and it is hence expected that they are strongly dominated by the contribution of a single fermion-loop (but nonperturbative in $\bar{g}$ and after renormalization). As a result one expects a rather linear dependence on $N_{f}$. Since the relevant effect for physics is the contribution of a single heavy quark, we rescale the relative mass effect as $\left(N_{f}=2\right)$

$$
\frac{1}{N_{f}} \frac{R(M)-R(\infty)}{R(\infty)}
$$

These numbers are listed in Table I for the ratios in Eq. (11).

Conclusions. - In conclusion, we pointed out the factorization formula Eq. (1) for the dominating dependence of low energy dimensionful quantities such as hadron masses on the mass of a heavy (dynamical) quark. In perturbation theory, the power law $P \sim(M / \Lambda)^{\eta_{0}}$ is a very good approximation and we find that the nonperturbative dependence is also rather close to that law for quark masses around $\frac{1}{2} M_{\mathrm{c}} \ldots \frac{1}{4} M_{\mathrm{c}}$. The knowledge of this mass dependence is expected to provide valuable information for tuning heavy quark masses to the correct point in future lattice QCD computations. We emphasize that our results are entirely sufficient to get the qualitative picture. At the quantitative level, they are limited to an accuracy of around $10 \%$, both because of the limited precision in the massless theory and because we have not yet taken a true continuum limit for the finite mass points in Fig. 1. At least the latter should be improved soon. In principle, one also has to worry about power corrections to the factorization formula, but Table I shows that these are irrelevant at the present level of precision.

The dominating effect in Fig. 1 originates from the mass dependence of the gauge coupling in the effective theory. It therefore disappears in dimensionless ratios of low energy scales at fixed mass $M$ and only leaves residual power law effects. The effective theory analysis predicts those to be of the form $M^{-2}$ for large $M$. Our investigation of these power corrections has been restricted to $M \leq \frac{1}{2} M_{\mathrm{c}}$.

TABLE I. Relative effects Eq. (13) for the ratios in Eq. (11). At $M_{\mathrm{c}}$ we quote the results from interpolations in $1 / M$ and $1 / M^{2}$;

\begin{tabular}{|c|c|c|c|c|c|c|}
\hline \multirow{2}{*}{$\begin{array}{l}M / \Lambda \rightarrow \\
R \\
\end{array}$} & \multicolumn{2}{|c|}{$M_{\mathrm{c}} / \Lambda$} & \multirow[b]{2}{*}{2.50} & \multirow[b]{2}{*}{1.28} & \multirow[b]{2}{*}{0.63} & \multirow[b]{2}{*}{0} \\
\hline & $1 / M$ scaled & $1 / M^{2}$ scaled & & & & \\
\hline$\sqrt{t_{0}} / w_{0}$ & $0.34(5) \%$ & $0.16(2) \%$ & $0.72(11) \%$ & $1.26(12) \%$ & $2.62(14) \%$ & $5.4 \%$ \\
\hline$\sqrt{t_{c} / t_{0}}$ & $0.28(3) \%$ & $0.13(1) \%$ & $0.59(6) \%$ & $1.06(3) \%$ & $1.74(3) \%$ & $3.2 \%$ \\
\hline $\begin{array}{l}r_{1} / r_{0} \\
r_{0} / \sqrt{t_{0}}\end{array}$ & $\begin{array}{l}0.45(13) \% \\
0.05(28) \%\end{array}$ & $\begin{array}{l}0.21(6) \% \\
0.02(12) \%\end{array}$ & $\begin{array}{l}1.0(3) \% \\
0.1(6) \%\end{array}$ & $\begin{array}{l}1.8(5) \% \\
0.7(5) \%\end{array}$ & $\begin{array}{l}2.6(6) \% \\
1.7(5) \%\end{array}$ & $\begin{array}{r}\approx 4.0 \% \\
3.0 \%\end{array}$ \\
\hline
\end{tabular}
see Fig. 2. 
Larger masses require smaller lattice spacings, larger lattices and (due to critical slowing down) larger statistics. However, in the accessible region we have precise results. Phenomenologically they are described by an approximate $M^{-1}$ law. We therefore interpolated between the largest simulated mass and the Yang-Mills theory to the charm mass as $M^{-n}$ with both $n=1$ and $n=2$. It seems safe to assume that the true results will be in between. In any case, the thus interpolated effects are very small, between 1 and 6 permille (Table I). This provides a message for today's dynamical fermion simulations. Dynamical charm effects are relevant only when one has very good precision, a very small lattice spacing and/or physical observables sensitive to higher energy scales.

We thank Hubert Simma and Ulli Wolff for their valuable comments on the manuscript and Martin Lüscher for a very useful discussion on the effective theory. We thank Andreas Athenodorou and Marina Marinkovic for their contributions in an early stage of this work. Simulations have been performed on the supercomputers Konrad and Gottfried at HLRN, Cheops at the University of Cologne (financed by the Deutsche Forschungsgemeinschaf), Stromboli at the University of Wuppertal and PAX at DESY, Zeuthen. We thank these institutions for support. This work is supported by the Deutsche Forschungsgemeinschaft in the SFB/TR 09 and the SFB/TR 55.

[1] Of course, in higher energy processes the heavier quarks play a relevant role, e.g., in the fundamental parameters of QCD for LHC physics, or, more generally, the $\Lambda$ parameter of the 5-flavor theory.

[2] T. Appelquist and J. Carazzone, Phys. Rev. D 11, 2856 (1975).

[3] S. Weinberg, Phys. Lett. 91B, 51 (1980).

[4] S. Weinberg, Physica (Amsterdam) 96A, 327 (1979).

[5] W. Bernreuther and W. Wetzel, Nucl. Phys. B197, 228 (1982); B513, 758 (1998).

[6] R. Sommer, Nucl. Phys. B411, 839 (1994).

[7] M. Lüscher, J. High Energy Phys. 08 (2010) 071.

[8] S. Borsanyi et al., J. High Energy Phys. 09 (2012) 010.

[9] We here use the language of a theoretical situation where all light quarks are massless. Light quark masses can be added with trivial changes, such as additional arguments in several functions.

[10] In regular schemes the couplings are related to, say, the $\overline{\mathrm{MS}}$ one by $\bar{g}^{2}=\bar{g}_{\overline{\mathrm{MS}}}^{2}+O\left(\bar{g}_{\overline{\mathrm{MS}}}^{4}\right)$.

[11] To be precise, we here assume that either $N_{l}=0$ where light quark fields are absent, or $N_{l} \geq 2$ where there is a nonanomalous chiral symmetry in the light sector. The statement also holds for theories with light quark masses if we define light-quark mass factors to be included in $\Phi_{i}(x)$. We thank Martin Lüscher for a clarification on the use of chiral symmetry in this context.

[12] Again we refer to the theoretical situation where the first $N_{l}$ flavors are massless; in general, also the light quark masses have to be matched.

[13] A. G. Grozin, M. Hoeschele, J. Hoff, and M. Steinhauser, J. High Energy Phys. 09 (2011) 066.

[14] K. Chetyrkin, J. H. Kühn, and C. Sturm, Nucl. Phys. B744, 121 (2006).

[15] A. Athenodorou, M. Bruno, J. Finkenrath, F. Knechtli, B. Leder, M. Marinkovic, and R. Sommer (to be published).

[16] T. van Ritbergen, J. A. M. Vermaseren, and S. A. Larin, Phys. Lett. B 400, 379 (1997).

[17] K. G. Chetyrkin, Phys. Lett. B 404, 161 (1997).

[18] J. A. M. Vermaseren, S. A. Larin, and T. van Ritbergen, Phys. Lett. B 405, 327 (1997).

[19] M. Czakon, Nucl. Phys. B710, 485 (2005).

[20] S. Capitani, M. Lüscher, R. Sommer, and H. Wittig (ALPHA Collaboration), Nucl. Phys. B544, 669 (1999).

[21] K. Jansen and R. Sommer (ALPHA Collaboration), Nucl. Phys. B530, 185 (1998).

[22] P. Fritzsch, F. Knechtli, B. Leder, M. Marinkovic, S. Schaefer, R. Sommer, and F. Virotta, Nucl. Phys. B865, 397 (2012).

[23] M. Marinkovic and S. Schaefer, Proc. Sci., LATTICE2010 (2010) 031 [arXiv:1011.0911].

[24] M. Lüscher and S. Schaefer, Comput. Phys. Commun. 184, 519 (2013).

[25] T. Korzec, https://github.com/to-ko/mesons.

[26] F. Knechtli, A. Athenodorou, M. Bruno, J. Finkenrath, B. Leder, M. Marinkovic, and R. Sommer, Proc. Sci., LATTICE2014 (2014) 288 [arXiv:1411.1239].

[27] K. Olive et al. (Particle Data Group), Chin. Phys. C 38, 090001 (2014).

[28] R. Sommer, Proc. Sci., LATTICE2013 (2014) 015 [arXiv: 1401.3270]. 